\title{
The impact and cost of child marriage prevention in three African settings-Brief
}

Population Council

Follow this and additional works at: https://knowledgecommons.popcouncil.org/departments_sbsr-pgy

Part of the Demography, Population, and Ecology Commons, Family, Life Course, and Society Commons, International Public Health Commons, and the Maternal and Child Health Commons How does access to this work benefit you? Let us know!

\section{Recommended Citation}

"The impact and cost of child marriage prevention in three African settings-Brief." Addis Ababa: Population Council, 2017. 


\section{THE IMPACT AND COST OF CHILD MARRIAGE PREVENTION IN THREE AFRICAN SETTINGS}

Child marriage affects a significant number of girls in developing countries. The negative consequences of the practice are both numerous and powerful, spanning health, welfare, development, and demographic domains. Yet there is limited evidence on what works to delay child marriage in different cultural contexts and even less information on programmatic cost. This study aims to identify the most effective and minimum basic package approaches to delay marriage among adolescent girls in different African settings. The study was undertaken in rural areas of Burkina Faso, Ethiopia, and Tanzania. The study also measures the cost of implementing different approaches, as well as cost-effectiveness. It provides information on how best to design programs that are scalable and sustainable within poor settings, and that can provide support for the millions of girls in developing countries at risk of child marriage.

\section{METHODOLOGY}

From 2010 to 2016, the Population Council and partners in Burkina Faso, Ethiopia, and Tanzania tested and costed interventions to delay the age at marriage. In each country, we implemented different approaches to address child marriage in different districts and measured the impact and cost of each approach. The approaches were:

- Community dialogue to encourage social norms change related to the practice of child marriage;

- Provision of school materials to encourage school attendance and thereby discourage an early, arranged marriage; and

- $\quad$ Provision of a conditional asset transfer in the form of livestock (a goat in Burkina Faso and Tanzania; chickens in Ethiopia), provided on the condition that girls remain unmarried and in school during the pilot program;

- $\quad$ Offering all three approaches in one district of each country, and

- A control area.

The impact of the interventions was measured through population-based surveys of adolescent girls at baseline, before the start of the interventions, and after roughly two years of implementation of the pilot approaches. In each country, 2,500 girls aged 12 to 17 were sampled, 500 per study cell, at each round of survey. The questionnaire used in the study was a structured instrument covering areas such as demographic characteristics and living conditions, education, families and parentchild relationships, livelihoods, attitudes toward marriage and the experience of marriage, sexual experience, family planning, maternal and child health, and HIV and AIDS. The study received ethical clearance from the Population Council's Institutional Review Board as well as local review boards in each of the study countries.
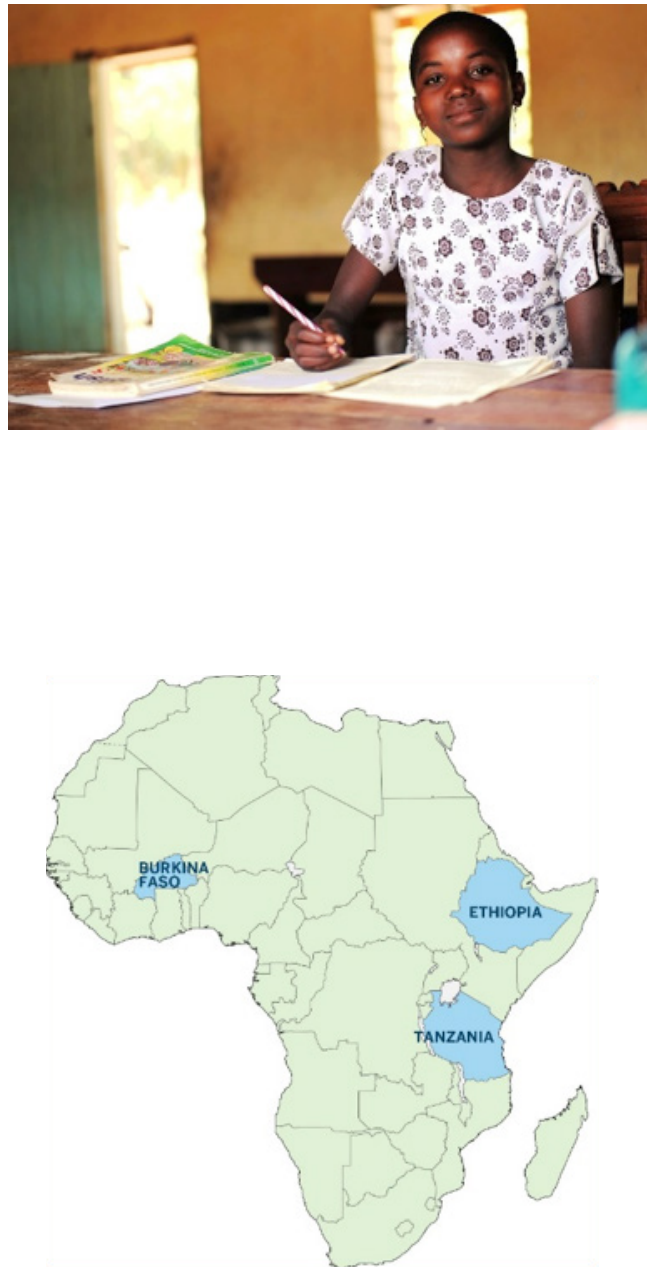


\section{ANALYSIS}

The outcome of the study is the percent of girls who have ever been married. Multivariate models controlled for age, socioeconomic status, ethnic group, and religion. Analysis was stratified by site and younger (age 12 to 14) and older (age 15 to 17) adolescents. In Burkina Faso and Tanzania, separate multivariate models are presented for each intervention site, with reference to the control. For these two countries, we present risk ratios (RRs) and 95\% confidence intervals for residence in the intervention sites versus the control site, at endline. For Ethiopia, unfortunately, the control site proved to be significantly different from the intervention sites because it was a resettlement area for people from other parts of the country, and the populations in this area were largely unfamiliar to each other. Marriage arrangements in Ethiopia are rooted in family ties and the desire to strengthen bonds between families who are known to each other and/or neighbors. As a result, we model the RR of being married at endline in each of the sites, with reference to the baseline. In addition, we did not assess the child marriage outcome for girls aged 12 to 14 in Burkina Faso, due to too few cases of marriage among this age group at both baseline and endline.

\section{RESULTS}

Table 1 shows the risk ratios for Burkinabe and Tanzanian girls having ever been married at endline, with reference to the control sites. In Burkina Faso, girls aged 15 to 17 residing in the community dialogue arm had over two-thirds less risk $(R R=0.33)$ of being married compared to those in the control site. In Burkina Faso, additional analysis demonstrated that the conditional asset site was significantly different from the control site at both baseline and endline, which was impossible to control for in the multivariate models. Thus, the conditional asset arm was eliminated from the analysis of results from Burkina Faso.

In Tanzania, the comprehensive model was significantly associated with a delay in marriage among girls 12 to 14 . Girls 12 to 14 residing in the comprehensive site had twothirds less risk of being married compared to girls in the control site $(R R=0.33)$. Tanzanian girls 15 to 17 residing in the conditional asset transfer site had roughly half the risk of being married compared to girls in the control site $(\mathrm{RR}=$ 0.52).

Prior to the account development phase, $\mathrm{MCl}$ conducted market research with adolescent girls who were in rural and urban areas, in school and out of school, and younger and older to determine their cash flows, their money management needs and their preferences for a savings product.
TABLE 1: Adjusted $^{1}$ risk ratios (95\% confidence intervals) for Burkinabe and Tanzanian girls having ever been married at endline, with reference to the control group, by study cell and age group

\begin{tabular}{|l|c|c|}
\hline \multicolumn{2}{|c|}{ Age 12 to 14 } & Age 15 to 17 \\
\hline BURKINA FASO & - & 0.33 \\
\hline Community dialogue & - & $0.19,0.60) * * *$ \\
\hline Education promotion & - & 0.99 \\
& $(0.66,1.49)$ \\
\hline “Full” model & - & 0.77 \\
& & $(0.52,1.15)$ \\
\hline TANZANIA & 1.13 & 0.74 \\
\hline Community dialogue & $(0.48,2.68)$ & $(0.43,1.27)$ \\
\hline Education promotion & 0.70 & 0.99 \\
& $(0.30,1.65)$ & $(0.64,1.53)$ \\
\hline Asset transfer & 0.41 & 0.52 \\
& $(0.15,1.15)$ & $(0.30,0.91) *$ \\
\hline “Full” model & 0.33 & 0.59 \\
& $(0.11,0.99) *$ & $(0.34,1.01)$ \\
\hline
\end{tabular}

Note: Weighted data. ${ }^{1}$ Adjusted for age, religion, ethnicity, and socioeconomic status.

${ }^{*} p<0.05 .{ }^{*} p<0.01 . * * * p<0.001$.

Among girls aged 12 to 14 in Ethiopia, those residing in the community dialogue site at endline had over half the risk of being married compared to their counterparts at baseline $(\mathrm{RR}=0.42)$. Girls 12 to 14 in the education promotion arm had 90 percent less risk of being married at endline compared to their counterparts at baseline (RR $=0.09)$. Among girls aged 15 to 17 , those residing in the conditional asset transfer arm and the comprehensive model had roughly half the risk of being married at endline compared to their counterparts at baseline ( $\mathrm{RR}=0.57$ for conditional asset transfer; RR $=0.38$ for comprehensive model).

\section{COST PER GIRL SERVED}

Table 2 shows the cost per girl served per year for the different intervention models. It should be noted that this is the cost to implement the activities and support girls through various approaches. Because the community dialogue model did not target girls alone, we estimated the magnitude of the population reached using population estimates and the percent of girls at endline who had heard about or participated in the social norms change activities.

Costs varied significantly between country and approach. In all countries, community dialogue and school promotion cost roughly the same: from $\$ 9$ to $\$ 20$ per community 
member served for community dialogue and from $\$ 13$ to $\$ 20$ per girl served with school supplies. The conditional asset transfer and the comprehensive models were the most costly to implement. In particular, provision of livestock and the comprehensive model were expensive in Tanzania, perhaps related to elaborate logistics required to purchase, store, and transport goats and to ensure the quality of the goats procured. Curiously, the full model in Ethiopia was cheaper to implement ( $\$ 29$ per girl per year) compared to the conditional asset transfer of two chickens per girl per year. Further examination of costing data suggested that fewer girls in the comprehensive arm received chickens at all rounds of the study, perhaps reflecting their failure to meet the project's criteria.

\section{CONCLUSION}

The study provided several valuable lessons for the design of child marriage prevention programs in sub-Saharan Africa.

\section{Simple, cost-effective interventions can be effective in delaying child marriage}

Research results found that simple, cost-effective interventions can be effective in addressing child marriage. Interventions that prove to be effective vary across contexts and the age group of the girl. Effective interventions for younger adolescents aged 12 to 14 may differ from those that are effective for older girls 15 to 17 , partly because the marriage market and community perceptions change once girls start getting older. Overall, we found that more economic interventions, such as conditional asset transfers, seemed to work for older adolescents, especially those in Tanzania and Ethiopia.

TABLE 2: Cost per girl/person served per year, by model and country

\begin{tabular}{|l|c|c|c|}
\hline & $\begin{array}{c}\text { BURKINA } \\
\text { FAS0 } \\
(n=3,235)\end{array}$ & $\begin{array}{c}\text { ETHIOPIA } \\
(n=5,167)\end{array}$ & $\begin{array}{c}\text { TANZANIA } \\
(n=4,154)\end{array}$ \\
\hline $\begin{array}{l}\text { Community } \\
\text { dialogue }\end{array}$ & $\$ 12$ & $\$ 20$ & $\$ 9$ \\
\hline $\begin{array}{l}\text { School } \\
\text { promotion }\end{array}$ & $\$ 13$ & $\$ 20$ & $\$ 18$ \\
\hline $\begin{array}{l}\text { Conditional } \\
\text { asset transfer }\end{array}$ & $\$ 33$ & $\$ 32$ & $\$ 107$ \\
\hline $\begin{array}{l}\text { Comprehen- } \\
\text { sive model }\end{array}$ & $\$ 60$ & $\$ 29$ & $\$ 117$ \\
\hline
\end{tabular}

It is necessary to identify true 'hotspots' for the intervention

Rates of child marriage are changing rapidly in some countries. In settings such as Amhara, Ethiopia, the practice is declining rapidly. In order to maximize investments in child marriage prevention, it is advisable to undertake initial rapid assessments to identify locations where the practice is most common. While DHS surveys may be important sources of information and guidance, we found that marriage markets are extremely localized and vary significantly at the subregional level, a level of estimation that DHS does not provide. For example, the initial country selection for this study included Kenya and Uganda, based on analysis of DHS data. Ultimately, we eliminated these two research sites, given the low levels of child marriage reflected in our baseline surveys. As such, rapid, community-level data collection is currently being undertaken by the Population Council and our partners to identify subregional locations with the highest prevalence of child marriage, in order to maximize investments in prevention.

\section{More complex models make it difficult to achieve coverage and quality of implementation}

Contrary to common assumptions, the more complex, comprehensive interventions addressing child marriage on a variety of levels were not necessarily more effective than the simple, single-approach models. This is partly due to the difficulty of effectively implementing complex models on the ground. Simpler approaches are less costly and still prove to be effective. We recommend implementation of these streamlined approaches in order to maximize the impact of child marriage investments.

\section{Attention to coverage of interventions at the population level}

In addition to the results presented here, we measured the population-level coverage of each of the interventions by going house-to-house to document the number of participants versus nonparticipants in the project areas. We found extreme variability in coverage of interventions, ranging from 20 to 91 percent. Program managers need to pay attention to coverage of interventions on the ground, ensuring that all eligible girls are offered participation. This is particularly important in rural communities where many sites are remote and inaccessible. 
Social norm change approaches should be systematic and intensive, not ad hoc

Community dialogue decreased risk of marriage in Ethiopia for girls aged 12 to 14 and in Burkina Faso for 15-17-yearolds. The approach to social norms change differed between project countries. In two countries-Burkina Faso and Ethiopia-the approach was an adapted version of the community conversations approach (developed and applied extensively in Ethiopia), whereby community members meet periodically with trained facilitators who take them through a systematic process of problem identification and problem-solving. In Tanzania, the social norms change approach was ad hoc, with community and religious leaders trained to deliver key messages at events they might host or attend. Only the systematic and intensive method of social norms change seemed to make an impact on the practice-as it did in Burkina Faso and Ethiopia-while informal delivery of messages, such as that undertaken in Tanzania, did not.

\section{Conditional asset transfers and supplies should be frequent and raise the visibility of girls}

The conditional asset arm was effective at delaying marriage among older girls in Ethiopia and Tanzania while incomparability between sites in Burkina Faso did not allow us to assess the effectiveness of this approach in that setting. Consistent with other reviews of child marriage prevention, economic interventions do seem to be effective in delaying the age of marriage. In Burkina Faso and Tanzania, asset transfer in the form of a goat was awarded once at the end of the intervention period (just over two years), while school supplies were given yearly. In Ethiopia, livestock in the form of chickens was provided on a yearly basis and school supplies were provided twice per year. More frequent provision of lower cost assets seems to have the effect of sustaining interest and commitment to the program. The conditional asset transfers scheme had lower participation in Burkina Faso and Tanzania, compared to Ethiopia. In addition, public, community-wide award of the commodities/prizes was used as a way to bring visibility to the status of girls in the project communities. The frequency of these events may have served to encourage girls and their parents to abide by the conditions. By comparison, participation in the asset transfer scheme was lower in Burkina Faso and Tanzania, despite the fact that a higher value goat was offered. The infrequency of awards may have resulted in fatigue and contributed to dropout.
Focus on out-of-school girls, family planning, and fertility awareness among girls who are at greatest risk of child marriage

Only a minority of girls were attending school at the time of their marriage: 43 percent in Ethiopia, 3 percent in Burkina Faso, and $<1$ percent in Tanzania. At the same time, many initiatives to prevent child marriage are implemented in the school environment, such as enforcement committees in the school where girls can report an impending marriage. Our data suggest that these approaches may be directed at low-risk, in-school girls. In settings where adolescents are sexually active outside of marriage, family planning services and information should be accessible. In all settings, knowledge of contraceptive, family planning services, and awareness of fertility should be promoted among adolescents. A focus on schooling, family planning, and fertility awareness can reduce vulnerabilities to child marriage.

\section{Limitations}

While we learned a number of lessons in our study, there were limitations. In Ethiopia we did not benefit from having a control site, due to the unfortunate selection of a resettlement area as the control location and inherent differences not captured by the survey. In Burkina Faso, we found that the conditional asset transfer site was incomparable to the control at both baseline and endline, even after controlling for background factors. This meant that we were unable to draw conclusions about the asset transfer approach in this country. Likewise, in Burkina Faso, the local language used in the survey is not commonly written. As such, the questionnaire remained in French and interviewers discussed and agreed upon the local language equivalent to be used in the survey. This may have introduced bias as it is possible that interviewers did not always recall the translation of questionnaire items and may have modified or distorted the translations.

\section{NEXT STEPS}

In Ethiopia and Burkina Faso, the Population Council and partners are undertaking scale-up of successful approaches in underserved areas. In Burkina Faso, community dialogue is being implemented in the Est region, while in Ethiopia, we are providing school supplies for younger girls and asset transfers for older girls in rural Beneshangul Gumuz region. 